\title{
ESTRATÉGIA DE VERIFICAÇÃO E VALIDAÇÃO EM CFD PARA ESCOAMENTO EM MEDIDOR DE VAZÃO
}

\author{
M. MUNIZ ${ }^{1}$, D. N. VENTURI ${ }^{1}$, L. STRECK ${ }^{1}$, V. R. WIGGERS ${ }^{1}$ e H. F. MEIER ${ }^{1}$ \\ ${ }^{1}$ Universidade Regional de Blumenau, Departamento de Engenharia Química \\ E-mail para contato: mmuniz_eq@ @otmail.com
}

\begin{abstract}
RESUMO - Realizou-se um estudo de verificação e validação por meio de experimentação física e numérica em um medidor de vazão do tipo Venturi. Os experimentos físicos foram realizados numa bancada experimental avaliando a diferença de pressão no medidor com um manômetro diferencial, e a vazão volumétrica de líquido por técnica gravimétrica, em treze condições operacionais. Os experimentos numéricos foram realizados via fluidodinâmica computacional em um código comercial, avaliandose dois modelos de turbulência, os efeitos geométricos e de refinamento de malha numérica. Para uma melhor comparação dos resultados, realizou-se também um estudo de quantificação de incerteza na medição da pressão e de vazão para os experimentos físicos, e uma avaliação da incerteza numérica com análise do índice de convergência de malha (GCI). A incerteza das medições físicas é de no máximo 2,7\%, nas vazões mais baixas, e a incerteza relacionada à malha é de no máximo $3 \%$.
\end{abstract}

\section{INTRODUÇÃO}

Equipamentos de medição de vazão, como o medidor Venturi, são utilizados tanto em escala industrial, quanto de bancada, devido sua simplicidade de construção e confiabilidade de medição.

No entanto, o projeto destes equipamentos baseia-se fortemente em correlações empíricas determinadas para vários casos específicos. Deste modo, a simulação computacional via técnicas de CFD destes dispositivos vem se tornando comum, no sentido de se avaliar parâmetros importantes antes de sua construção, poupando gastos financeiros e aumentando a confiabilidade do equipamento (PALADINO, 2005).

Para uma comparação precisa entre as informações obtidas nos experimentos físicos e numéricos, é importante que se realize a quantificação das incertezas respectivas.

Nas medições físicas, as incertezas de medição podem ser calculadas por propagação de erros, através das equações nas quais as grandezas são determinadas, ou por tratamento estatístico. No primeiro caso, estes erros devem ser conhecidos à priori, e podem vir de informações do fabricante sobre um equipamento de medida.

No caso das incertezas numéricas em fluidodinâmica computacional, Freitas (2002) as divide 
em três grupos principais: associada aos dados de entrada; associada ao modelo; e associada à solução das equações.

Quanto ao primeiro tipo, pôde-se reduzi-la a níveis quase nulos, obtendo bons valores para as propriedades físicas do fluido e definindo corretamente as condições de contorno. No caso do segundo tipo, pode-se concluir sobre esta incerteza após avaliar diferentes modelos e compará-los, à posteriori, com dados experimentais. Já o terceiro tipo, provém dos erros de discretização espacial e temporal e dos erros iterativos, e para quantificação deste tipo de erro, pode-se utilizar o método GCI, proposto por Roache (1994), baseado na extrapolação de Richardson.

Neste trabalho, uma comparação entre dados experimentais e dados simulados é realizada, a fim de que se valide um modelo tridimensional para a simulação destes medidores. Outra contribuição deste trabalho é o estudo da influência da forma como a medição da pressão é realizada na simulação numérica, para que seja mais condizente com a realidade experimental.

\section{MATERIAIS E MÉTODOS}

\subsection{Experimentação Física}

A bancada utilizada para a obtenção dos dados experimentais opera com água, e possui sistema de controle e aquisição de dados acoplado ao sensor de medição de pressão instalado no tubo Venturi. As dimensões, em milímetros, do medidor estão discriminadas na Figura 1. A tubulação se estende por $15 \mathrm{D}$ à montante e $14 \mathrm{D}$ à jusante. $\mathrm{O}$ manômetro diferencial instalado com saída de 4 - 20mA no aparato, tem faixa de medição entre 0 e $1000 \mathrm{mmCa}$, com incerteza de $\pm 5 \mathrm{mmCa}$, fornecida pelo fabricante.

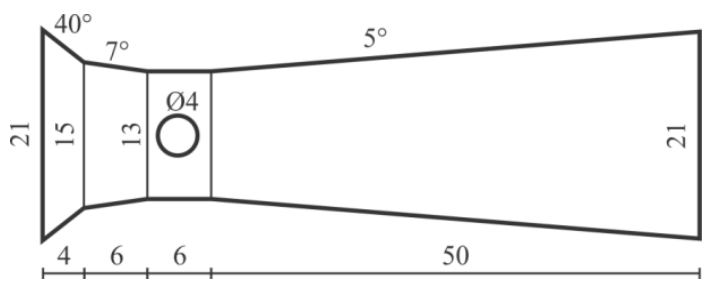

Figura 1 - Dimensões (em milímetros) do medidor Venturi.

Curva de Calibração: Para o início da obtenção de dados experimentais na bancada, o sensor de pressão deve ser calibrado manualmente, alterando os parâmetros de zero e span, até que meçam corretamente o valor de pressão referente à uma coluna de água. Em seguida, um valor de diferença de pressão é definido para o medidor Venturi, e o controle da unidade atua na rotação da bomba para que este valor seja satisfeito. Após a estabilização do escoamento, fase em que o desvio padrão da pressão medida é insignificante, a água é coletada em um recipiente plástico, e a razão entre a massa coletada (medida por uma balança) e o tempo de coleta (medido por um cronômetro digital) resulta na vazão mássica. Para o cálculo da vazão volumétrica, assume-se massa específica da água igual a $998,2 \mathrm{~kg} / \mathrm{m}^{3}$. 
Os experimentos de gravimetria foram realizados em quadruplicata, com treze valores de diferença de pressão contempladas no intervalo entre 100 e $700 \mathrm{mmCa}$. O valor adotado como vazão experimental é a média aritmética dos quatro experimentos.

Uma forma convencional de se representar a relação entre vazão volumétrica e diferença de pressão para um medidor Venturi, é através de um balanço de quantidade de movimento entre os pontos de medição, que pode ser escrito como:

$$
Q_{\text {ideal }}=A_{2} \sqrt{\frac{2 \Delta P}{\rho\left[1-\left(\frac{D_{2}}{D_{1}}\right)^{4}\right]}}
$$

Uma relação linear pode ser estabelecida entre a vazão experimental e a vazão ideal, com coeficiente linear nulo e coeficiente angular igual à $C_{d}$, que é um coeficiente de correção decorrente das forças que não se levam em conta na dedução anterior, como o atrito.

No presente estudo, a incerteza na variável da ordenada $\left(Q_{\text {real }}\right)$ é estimada pelo desvio padrão, uma vez que os experimentos foram realizados de forma manual e é difícil se quantificar o erro introduzido pela manipulação humana, juntamente com os erros da balança e do cronômetro. Já, para as incertezas na variável da abscissa $\left(Q_{\text {ideal }}\right)$, pode-se deduzir uma expressão a partir das Equações (1) e $(2$.

Para determinar a propagação de incertezas nos cálculos, Taylor (2012) mostra que para uma função genérica $q(x, \cdots, z)$, sua incerteza $\delta q$ é função das derivadas parciais de suas variáveis independentes, desde que todos os erros sejam independentes e aleatórios:

$$
\delta q=\sqrt{\left(\frac{d q}{d x} \delta x\right)^{2}+\cdots+\left(\frac{d q}{d z} \delta z\right)^{2}}
$$

No ajuste do coeficiente angular foi utilizado o método dos mínimos quadrados ponderado, o qual preconiza que cada par ordenado $(x, y)$ está sujeito à uma incerteza diferente e apenas na variável do eixo y (uma incerteza equivalente, neste caso). Onde esta ponderação é feita a partir do peso calculado na Equação (3, dada por Taylor (2012). Também é possível calcular a incerteza no valor no coeficiente angular $C_{d}$, pela Equação (4.

$$
\begin{aligned}
& w=\frac{1}{\delta_{y, \text { equiv }}^{2}}=\frac{1}{\left(\delta_{y}\right)^{2}+\left(C_{d} \delta_{x}\right)^{2}} \\
& \delta_{C d}=\frac{1}{\sqrt{\sum w x^{2}}}
\end{aligned}
$$




\subsection{Experimentação Numérica}

Modelagem matemática: Um modelo tridimensional, monofásico, isotérmico, em regime estacionário, e com propriedades físicas constantes foi proposto para as simulações, utilizando a abordagem euleriana. Também, foram utilizados dois modelos de turbulência: o k- $\varepsilon$ padrão, modelo de duas equações; e o RSM (Reynold Stress Model) padrão, modelo de sete equações (FERZIGER; PERIĆ, 2002).

Geometria: Duas geometrias foram desenhadas para representar o medidor Venturi: na primeira, geometria A (Figura 2a), se desenhou somente a tubulação principal e o estrangulamento; e na segunda, geometria B (Figura 2b), foram desenhados também as mangueiras para medição da pressão, similares aos existentes na experimentação física, que ligam o medidor à célula de carga. Em ambos os casos, a tubulação à montante possui 10D e à jusante possui 5D.

Simulação numérica: Para a simulação numérica, utilizou-se um código comercial que aplica o método dos volumes finitos, e para isto, as malhas hexaédricas detalhadas na Tabela 1 foram utilizadas. A condição de velocidade de entrada foi variada em 13 valores, utilizando para cada geometria as três malhas, e os dois modelos de turbulência. Ao final, os resultados foram analisados da seguinte forma (conforme a Figura 2):

a) medindo a pressão média no plano;

b) medindo a pressão pontual na parede;

c) medindo a pressão média na face superior dos tubos de medição (apenas para a geometria B).

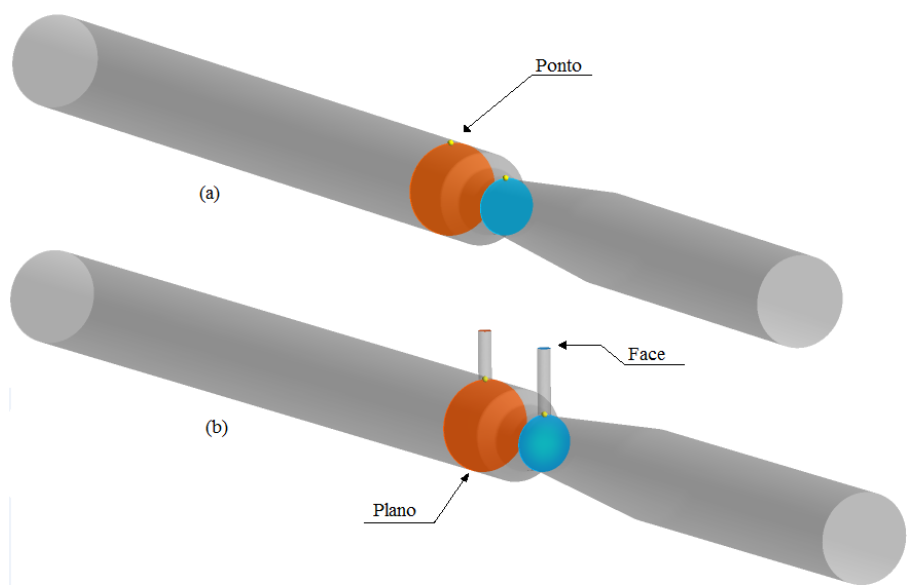

Figura 2 - Geometrias utilizadas nas simulações. (a) sem tubos, e (b) com tubos de medição.

Quantificação da incerteza: $\mathrm{O}$ estudo da incerteza relacionada à malha numérica foi realizado utilizando a metodologia GCI. O procedimento utilizado neste trabalho é apresentado em PROCEDURE..., 2008. Para isto, três malhas são necessárias, com razão de refinamento entre elas acima de 1,3 para garantir que o erro de discretização seja diferenciado dos outros. 
Tabela 1 - Parâmetros das malhas numéricas utilizadas para a análise de incerteza

\begin{tabular}{|c|c|c|}
\hline Parâmetro & Geometria A & Geometria B \\
\hline$N_{1}$ & 106.904 & 148.368 \\
\hline$N_{2}$ & 297.013 & 467.590 \\
\hline$N_{3}$ & 829.785 & 1.151 .867 \\
\hline$r_{21}$ & 1,41 & 1,35 \\
\hline$r_{32}$ & 1,41 & 1,47 \\
\hline
\end{tabular}

\section{RESULTADOS}

A partir da experimentação numérica, o valor determinado para $C_{d}$ foi de $0,97564 \pm 0,00215$. Este valor se aproxima com o estimado por Martins (1998), onde o coeficiente de descarga para este

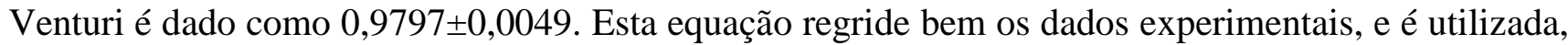
a partir deste ponto, para comparação com os dados numéricos.

Neste trabalho foram realizadas 390 simulações numéricas (2 medições para a geometria A e 3 para a B; 2 modelos de turbulência; 3 malhas numéricas; 13 condições operacionais) para que se pudesse extrair o máximo de informações acerca do comportamento da diferença de pressão nos diferentes casos, e a dependência da incerteza com cada um.

Uma vez que a geometria que mais representa o experimento real é a geometria $\mathrm{B}$, com medição de pressão na face dos manômetros virtuais, é apresentada, a seguir, a análise de GCI para este caso. A Figura 3 apresenta a evolução da diferença de pressão no medidor de vazão com o refino da malha numérica, e os valores para a extrapolação do método, que caracterizaria uma malha infinita (solução exata). Também, a figura mostra a incerteza relacionada à discretização espacial em cada condição operacional. Nota-se que neste caso a incerteza máxima é da ordem de 1,1\% e, a mínima, de 0,4\%.

A Figura 4 compara os dados experimentais e os dados numéricos simulados para as duas geometrias, com a malha mais refinada e modelo k- $\varepsilon$. A hachura na linha contínua representa a incerteza física da vazão, e as barras de erro nos pontos numéricos representam a incerteza calculada pelo método GCI. A área da figura foi limitada a duas condições operacionais para facilitar a visualização, porém são representativos do todo, já que os demais pontos tem comportamento similar. Pode-se observar que, nas duas geometrias, a medição da pressão no plano está em conformidade com os dados experimentais, enquanto a medição no ponto está em desacordo. Nota-se, também, que a medição na face (geometria B) também pode ser dita como de acordo, já que as regiões de incerteza se interceptam. Para o modelo RSM, o comportamento das variáveis é similar.

Já a Figura 5 mostra apenas a comparação dos dados experimentais com os modelos k-є e RSM, para malha refinada do caso mais simples: geometria A; e medição no plano. Uma vez que esta condição se mostrou satisfatória nas análises anteriores. Pode-se perceber que os dois modelos são satisfatórios para a predição da diferença de pressão do equipamento, e, como o modelo k- $\varepsilon$ adiciona menos equações de conservação ao sistema, frente ao modelo RSM, o primeiro acaba se tornando uma escolha mais razoável. 
No entanto, a comparação dos dados experimentais com os modelos k- $\varepsilon$ e RSM, para a malha refinada da geometria $\mathrm{B}$, com medição na face, na Figura 6, mostra que a utilização da geometria mais próxima da real, e de um modelo de turbulência anisotrópico, com a medição virtual igual à medição física, aproxima cada vez mais o valor numérico da diferença de pressão no Venturi, do valor real.
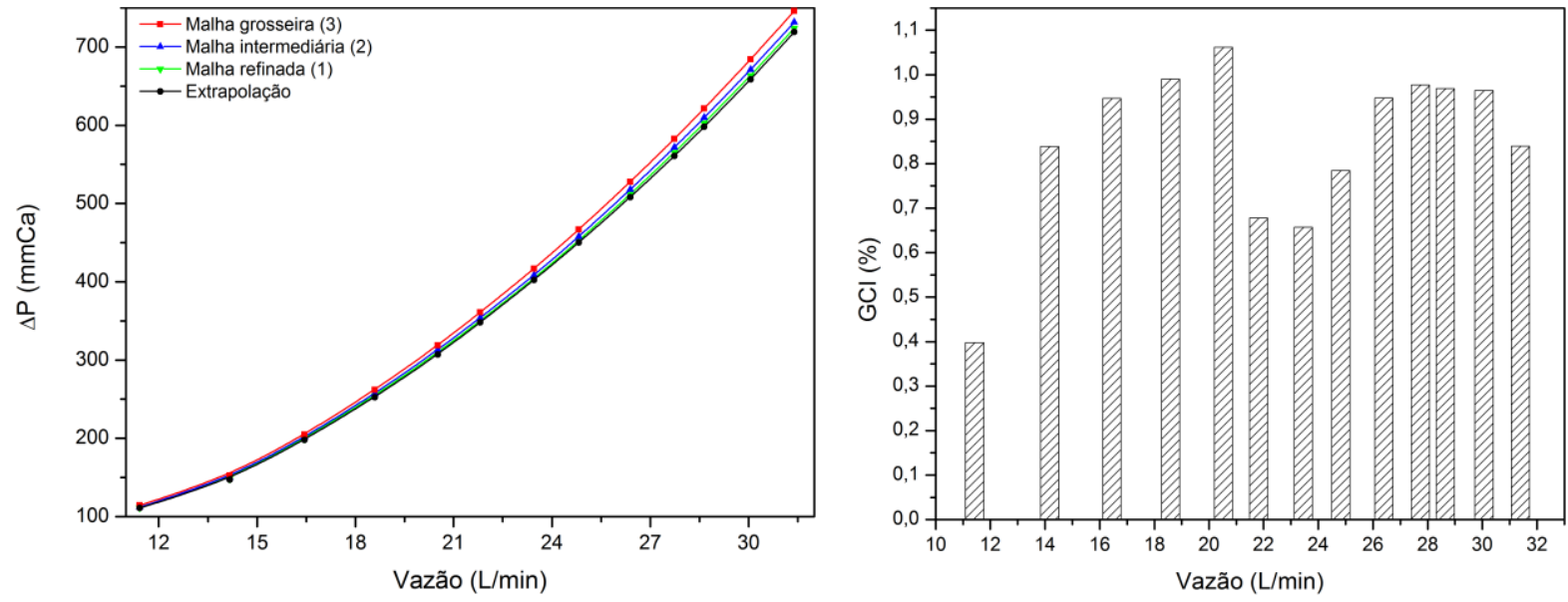

Figura 3 - Análise de GCI para a simulação numérica da geometria B, com modelo de turbulência k$\varepsilon$, e medição de pressão na face. À esquerda, a diferença de pressão como função do refinamento da malha, e à direita, a incerteza.

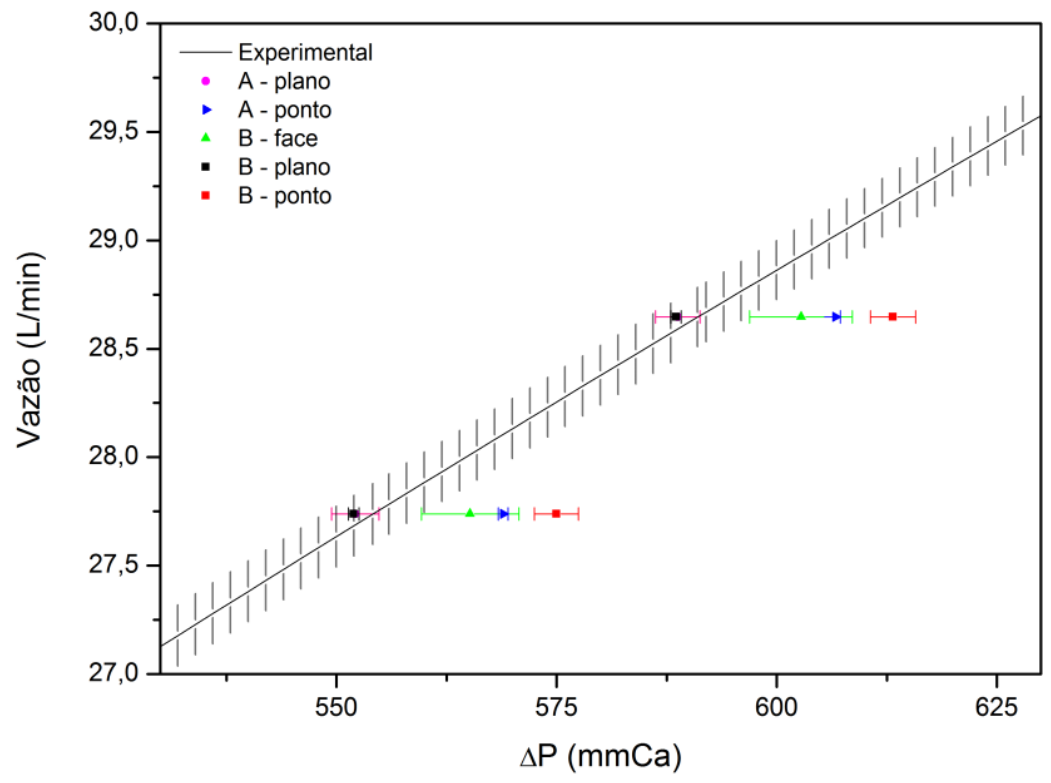

Figura 4 - Vazão como função da diferença de pressão no medidor Venturi para os experimentos físicos e numéricos (simulados com a malha refinada e modelo k- $\varepsilon$ ), e suas respectivas incertezas. 


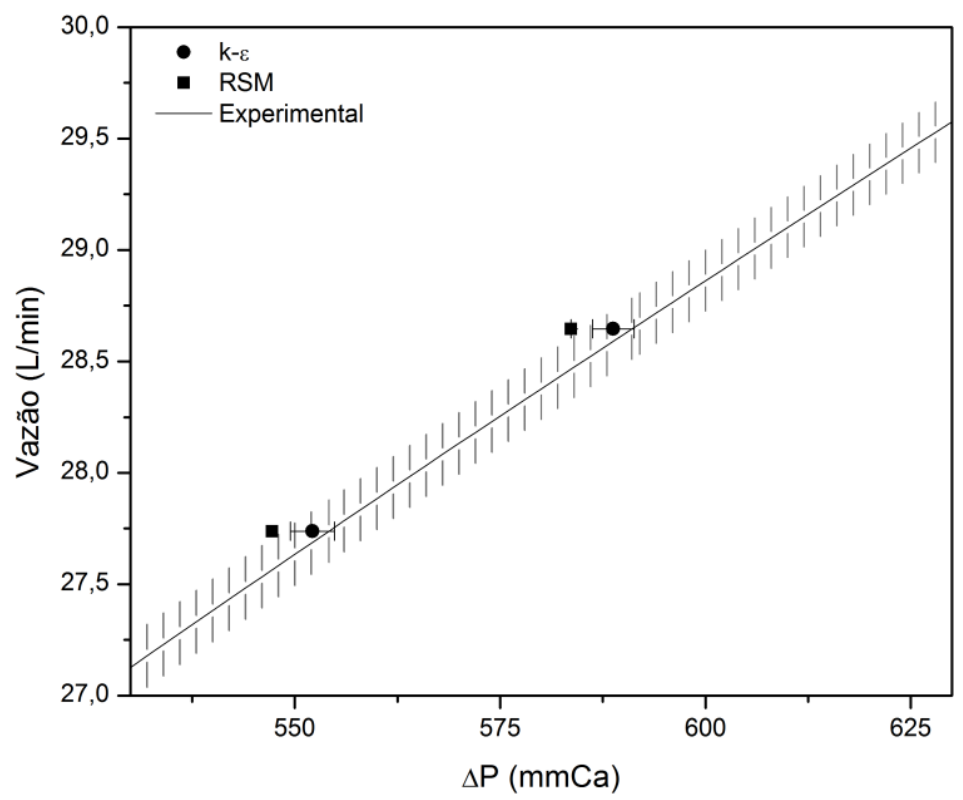

Figura 5 - Comparação dos dados experimentais com os modelos k- $\varepsilon$ e RSM, para malha refinada, geometria A e medição no plano, com suas respectivas incertezas.

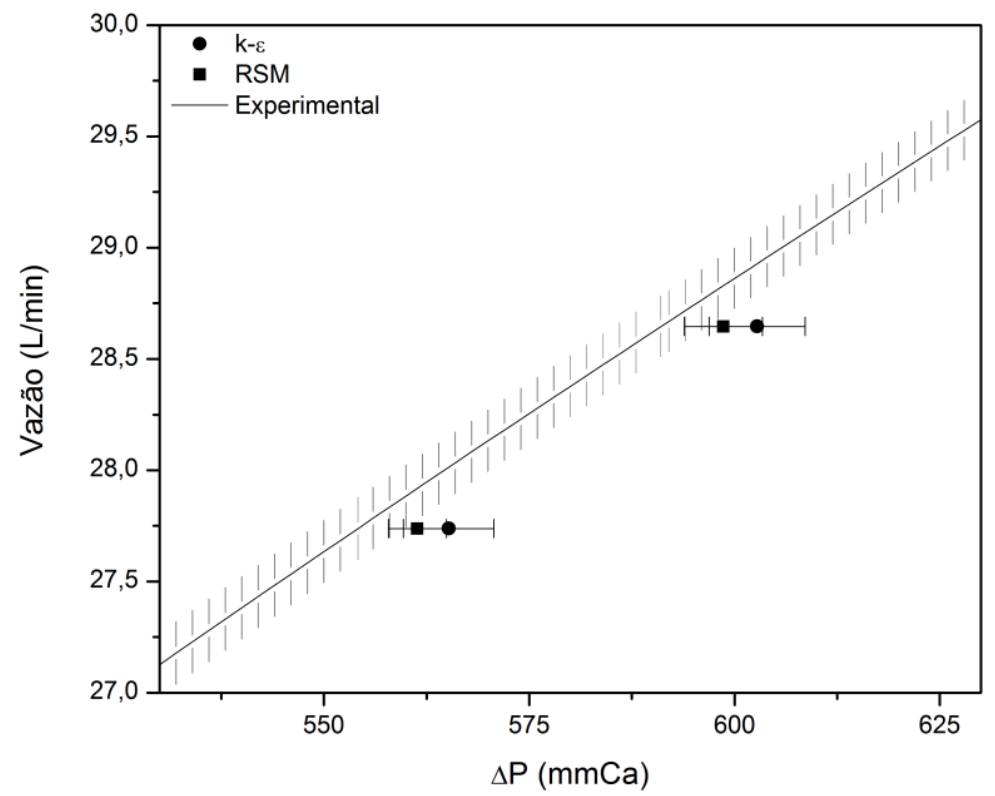

Figura 6 - Comparação dos dados experimentais com os modelos k- $\varepsilon$ e RSM, para malha refinada, geometria B e medição na face, com suas respectivas incertezas. 


\section{CONCLUSÃO}

Os resultados apresentados aqui mostram que uma análise de incerteza acoplada, entre os experimentos físicos e numéricos, é fundamental para a validação de modelos na área da fluidodinâmica computacional. Não é possível se ter segurança nas avaliações, sem uma análise consistente dos erros associados às técnicas utilizadas, principalmente porque se percebeu que a incerteza é função da vazão, no caso físico, e função da geometria, vazão e modelo de turbulência, no caso numérico.

Conclui-se, também, que o método de calibração utilizado para o experimento físico é confiável, apresentando incerteza alta apenas nas vazões próximas de zero. A incerteza da vazão, na faixa de trabalho do equipamento, é da ordem de $2,7 \%$ na vazão de $10 \mathrm{~L} / \mathrm{min}$, e de $0,4 \%$ em $30 \mathrm{~L} / \mathrm{min}$.

Ainda, ambos os modelos, k- $\varepsilon$ e RSM são adequados para a avaliação da diferença de pressão em um medidor de vazão do tipo Venturi, e a incerteza máxima relacionada à malha é de 3\% (para a malha refinada), considerando todos os casos simulados.

\section{NOMENCLATURA}

$A_{2} \quad$ área de estrangulamento $\left(\mathrm{m}^{2}\right)$

$\Delta P \quad$ perda de carga $(\mathrm{Pa})$

$C_{d} \quad$ coeficiente de descarga

w peso no ajuste dos mínimos quadrados

$D_{1} \quad$ diâmetro de entrada (m)

$D_{2}$ diâmetro de estrangulamento (m)

$Q \quad$ vazão volumétrica $\left(\mathrm{m}^{3} / \mathrm{s}\right)$

$\delta \quad$ incerteza da variável

$\rho \quad$ massa específica $\left(\mathrm{kg} / \mathrm{m}^{3}\right)$

\section{REFERÊNCIAS}

FERZIGER, J. H.; PERIĆ, M. Computational methods for fluid dynamics. 3. ed. Berlin; etc.: Springer, 2002.

FREITAS, C. J. The Issue of Numerical Uncertainty. Appl. Math. Model., v. 26, p. 237-248, 2002.

MARTINS, N. Manual de medição de vazão: através de placas de orifício, bocais e venturis. Rio de Janeiro: Interciência: Petrobras, 1998.

PALADINO, E. E. Estudo do escoamento multifásico em medidores de vazão do tipo pressão diferencial. Tese (Doutorado em Engenharia Mecânica) - UFSC, Florianópolis, 2005.

PROCEDURE for estimation and reporting of uncertainty due to discretization in CFD applications. J. Fluids Eng., v. 130, 2008.

ROACHE, P. J. Perspective: A method for uniform reporting of grid refinement studies. J. Fluids Eng., v. 106, p. 405-413, 1994.

TAYLOR, J. R. Introdução à análise de erros: o estudo de incertezas em medições físicas. 2. ed. Porto Alegre: Bookman, 2012. 